\title{
Opponent Color Processing Based on Neural Models
}

\author{
Maik Bollmann, Bärbel Mertsching \\ University of Hamburg, Department of Computer Science, Section IMA \\ Vogt-Kölln-Str. 30, 22527 Hamburg, Germany \\ e-mail: bollmann@ or mertsching@informatik.uni-hamburg.de \\ URL: http://ima-www.informatik.uni-hamburg.de/
}

\begin{abstract}
In this paper we present a new opponent color system which imitates some of the known color processing neural cells established by electrophysiological recordings. We describe the benefits of this system to image processing tasks. The opponent color model is embedded in an active vision system to improve the systems fixation and recognition capabilities. This is done by removing illumination effects to some degree and by evaluating the resulting color differences. Experimental results are presented.
\end{abstract}

\section{Introduction}

The idea of the existence of opponent colors has a long history beginning with Leonardo da Vinci and Goethe. The theory of opponent colors was derived from the polar structure of color perception. Originally it was based on psychophysical experiments. Hering [Hering 1878, 1880] developed the first opponent color theory due to color perception phenomena which could not be explained by the classical trichromatic color models of color mixture [Young 1802; Helmholtz 1867]. One of those phenomena is the simultaneous contrast.

Four unique colors located on opposite directions of the hue circle, the pairs red-green and blue-yellow, characterize an opponent color system. Thus colors between two unitary hues are of intermediate line. The color space is completed by a third pair (black-white) to describe saturation and intensity changes of colors.

Hurvich and Jameson [Hurvich 1955; Jameson 1955, 1956] advanced the opponent color theory by conducting systematic psychophysical experiments in order to explain many known color phenomena. The appropriateness of the trichromacy at the level of the retinal cones and the opponent color theory on higher levels of the visual path could first conclusively be shown by neurophysiological research. Different opponent color cell types which receive their input from the cones were found in the retina, in the LGN (lateral geniculate nucleus), and in the visual cortex (see e.g. [Wiesel 1966; Hubel 1968; Zeki 1973; Gouras 1981]).

Buchsbaum and Gottschalk [Buchsbaum 1983] have shown that efficient information transmission is achieved by opponent color coding. Usui et al. [Usui 1991, 1994] trained neural network models by means of a backpropagation algorithm. The hidden 
units learned characteristics similar to those of opponent color cells known from neurobiology. Another opponent color system is the ATD model of Guth [Guth 1991, 1993] which provides an uniform chromaticy space and predicts perceptions of colored light. Yamaba and Miyake [Yamaba 1993] developed a color character recognition system based on a modified opponent color model. Nevertheless, there are only a few applications of opponent color systems for image processing and recognition tasks.

We introduce a new opponent color system which extends the Neural-Active-VisionSystem NAVIS by processing the information 'color'.

\section{The Neural-Active-Vision-System NAVIS}

NAVIS (see [Drüe 1994]) has been developed for exploring and tolerant object recognition tasks in real world scenes. At present it is not restricted to applications in a certain domain.

It can roughly be separated into four functional components which are all based on neurobiological models: Fixation, feature extraction, invariance, and recognition. The color information is processed in two opponent color channels which are part of the feature extraction. The segmented colored regions are used in the fixation module and the associative layers of the recognition unit.

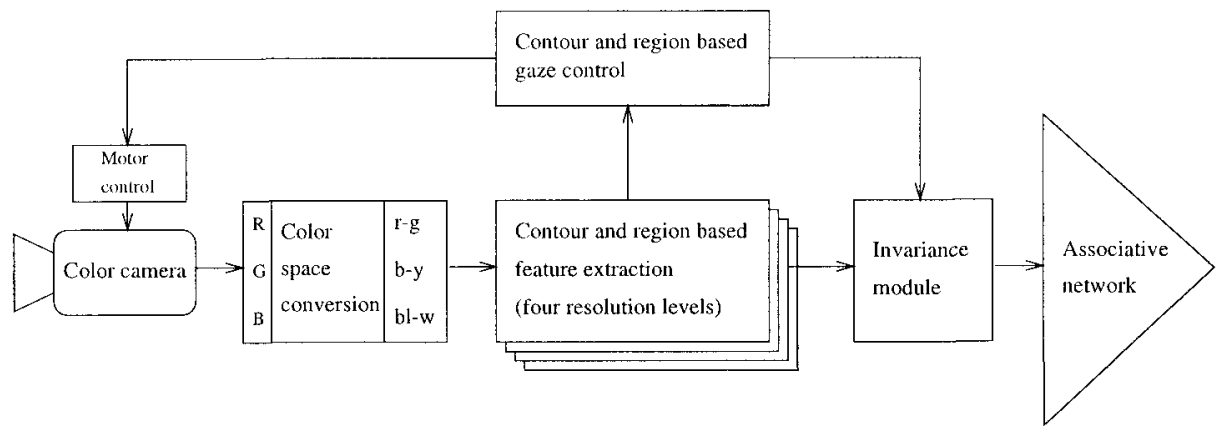

Fig. 2.1 Simplified block diagram of NAVIS

NAVIS explores its environment with a binocular camera system and learns the objects in its visual field in order to recognize them later in any arbitrary position. For this task it requires a high degree of adaption to changes in illumination. Our opponent color model described in the next paragraph shows approximate color constancy (see also [Bollmann 1995]). 


\section{The Opponent Color Model}

Human beings perceive colored objects in their visual field with the help of their three classes of retinal cones which differ in the spectral response curves while absorbing light being reflected from the objects in the environment or emitted by light sources. This first stage in the visual perception corresponds to the three channels of the CCD camera used in NAVIS.

The simple opponent color cells of the retina and the LGN form the second stage of color processing along the human visual path. According to the classification of Hubel and Wiesel there are two major classes of opponent color cells, the type-I-cells and the type-II-cells. The type-I-cells possess receptive fields which reveal a centersurround behavior while the type-II-cells show a simple color antagonism. The type-Icells can be subdivided into cells excited by increments of light in the center of their receptive field (on-center), and those excited by decrements of light in their center (off-center). They are further subdivided into cells whose centers get input from the $\mathrm{R}$ or $\mathrm{G}$ cones and those getting input from the B cones. In our technical system, the opponent color layer consists only of models of the $\mathrm{r}^{+} \mathrm{g}$-cells (red on-center/green offsurround type-I-cells) and the $\mathrm{b}^{+} \mathrm{y}^{-}$-cells (blue on-center/yellow off-surround type-Icells). The number of color channels had to be restricted in NAVIS due to runtime considerations.

The biological $\mathrm{r}^{+} \mathrm{g}$-cells receive their input from the red and green cones of the retina. Hence, the model cells of this type obtain data from the R and $G$ band of the RGB input image. The receptive field of the biological $b^{+} y^{-}$-cells is formed by blue cones on the one side and by a combination of red and green cones on the other side. In our model, this cell type gets input from the grabbed B band and from a computed yellow image where each pixel of the yellow image gets the minimum of the corresponding pixels of the $R$ and $G$ band.

The receptive field of an opponent color cell can be separated into a center and a surround system. If we assume these fields as circularly symmetric they can be described by Gaussian functions (equation 3.1). The parameters $\left(c_{1}, c_{2}, \sigma_{1}, \sigma_{2}\right)$ of these functions were determined heuristically and depend on the resolution levels or the kernel sizes respectively. Taking into account that the integral of the sum of the two Gaussians has to be zero, our model cells show no activity when illuminated with white light. The described receptive fields are realized as two-dimensional discrete convolution kernels (fig. 3.1). The discrete values of the kernel elements correspond to the synaptic weights of ganglion cells.

$$
W_{\text {center }}(x, y)=c_{1} \cdot e^{-\frac{x^{2}+y^{2}}{\sigma_{1}}}, W_{\text {surr }}(x, y)=-c_{2} \cdot e^{-\frac{x^{2}+y^{2}}{\sigma_{2}}}, \sigma_{1}<\sigma_{2}
$$




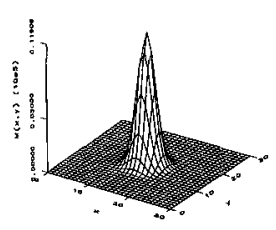

center system

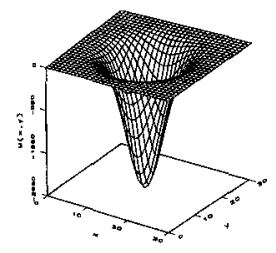

surround system

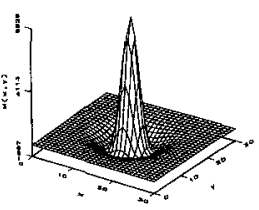

combined system

Fig. 3.1 Models of the receptive fields

The result of the calculation of the netto cell input (equation 3.2) is weighted by a tangenshyperbolicus mapping function (equation 3.3) which models the response of biological neurons. The parameters $m$ and net $t_{\text {off }}$ are determined in such a way that the cells respond with maximum activity to color differences larger than 200 steps. This output function improves the segmentation of colored regions and provides color constancy to a certain degree. The block diagram in fig. 3.2 illustrates the color processing steps in NAVIS.

$$
\operatorname{net}_{i}(x, y)=\sum_{k=0}^{K} \sum_{l=0}^{L} w_{k, l}^{\text {center }} \cdot p_{x-K / 2+k, y-L / 2+l}+w_{k, l}^{\text {surr. }} \cdot q_{x-K / 2+k, y-L / 2+l}
$$

$$
G\left(\text { net }_{i}\right)=\frac{1}{2} \cdot\left[\tanh \left(m \cdot\left(\text { net }_{i}-\text { net }_{\text {off }}\right)\right)+1\right]
$$

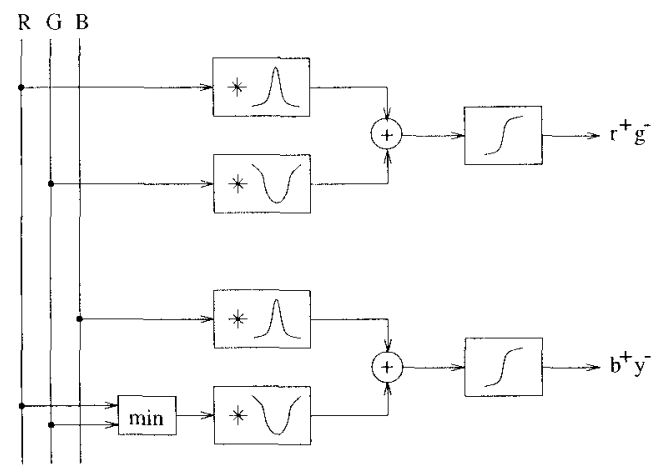

Fig. 3.2 Block diagram of the opponent color system 
The images presented to NAVIS pass through the opponent color layer which processes them as described. The resulting activities of the opponent color cells will be interpreted in the fixation and the pattern recognition unit of NAVIS as described in section 5 .

\section{Experimental results}

To estimate the illumination independence achieved by our system synthetic image material was produced. Also, we added different illumination gradients to all the three bands of Mondrian-like images. Fig. 4.1a shows the line profiles of an arbitrary row selected from the $\mathrm{R}$ band for two different gradients (logarithmic scale). Fig 4.1b depicts the corresponding output line profiles of the red-green channel.

a)
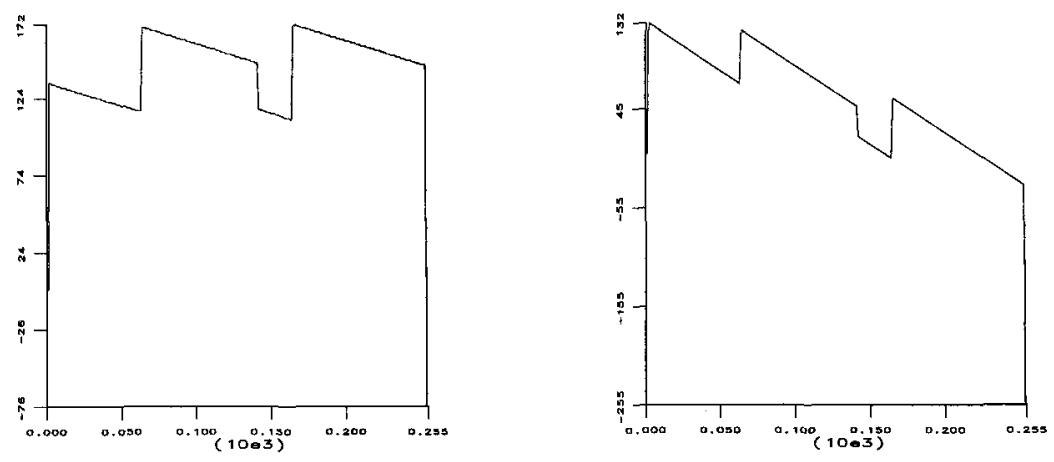

b)
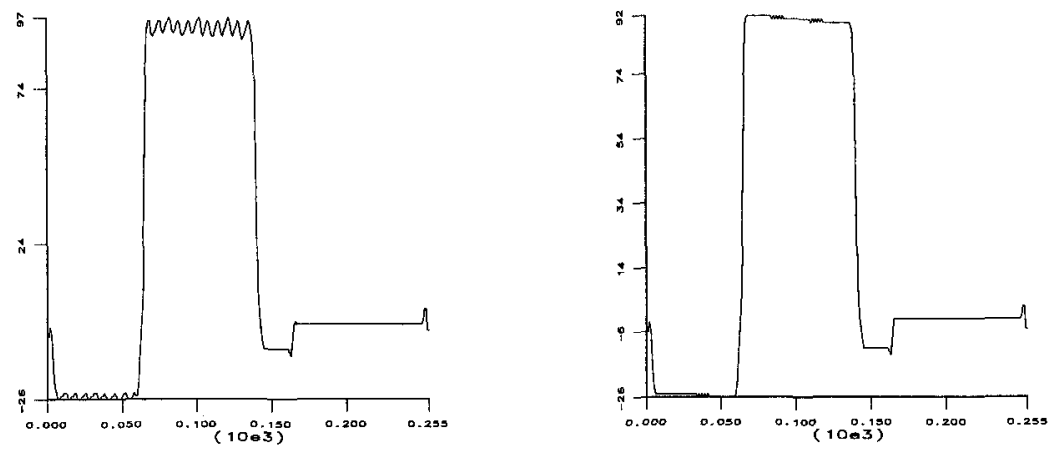

Fig. 4.1 a) Line profiles of two Mondrian-like input images

b) Line profiles of the corresponding output images of the opponent color layer 
The opponent color channels yield qualitatively similar images for both gradients. The intensity functions are widely constant for homogenous colored patches. This result corresponds well with results aimed at by the retinex theorie of Land [Land 1971, 1983, 1986] and the lightness algorithm of Horn [Horn 1974, 1986].

The following pictures show the segmentation characteristics of the opponent color model. It is designed that the primary hues red, green, blue, and yellow stimulate only one cell type in every case. This way the hues can easily be classified. Fig. $4.2 \mathrm{c}$ and fig. $4.2 \mathrm{~d}$ depict the segmentation outcome of two different computations of the yellow image required for the blue-yellow channel. The former is achieved by the proposed minimum method while the latter results from the frequently reported arithmetic averaging of the $R$ and $G$ band. In fig. $4.2 \mathrm{~d}$ the $\mathrm{b}^{+} \mathrm{y}^{-}$-cells are strongly inhibited by the red nose of the mandril which is obviously not yellow. Thus, the minimum function leads to a more perceptually correct processing.

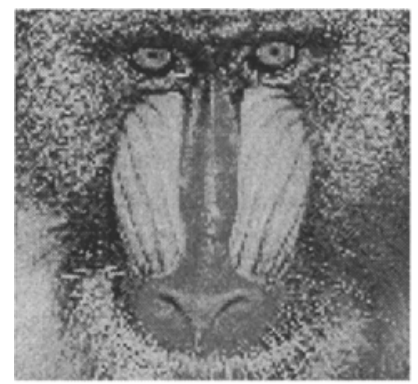

a) RGB input image

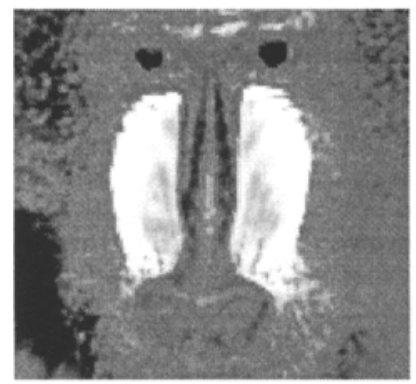

c) activities of the $b^{+} y^{*}$-cells; $y=\min (r, g)$

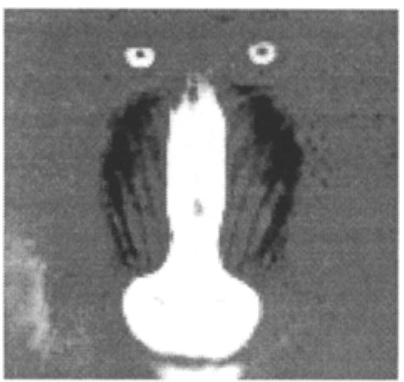

b) activities of the $\mathrm{r}^{+} \mathrm{g}^{-}$-cells

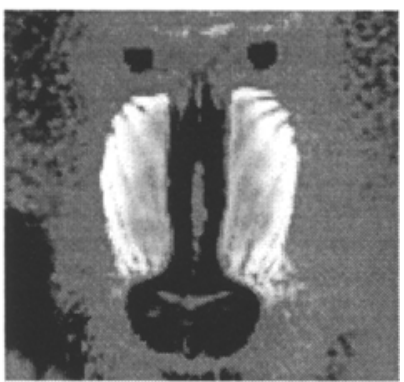

d) activities of the $b^{+} y^{*}$-cells;

$$
\mathrm{y}=0.5 \cdot(\mathrm{r}+\mathrm{g})
$$

Fig. 4.2 Segmentation results of the opponent color model ( 8 bit resolution):

Excited cells range from white to midgray while inhibited cells are shown in midgray to black. Midgray encodes no cell activity. 


\section{Benefits of the opponent color system}

The activities of the opponent color cells represent region features which can be used in the fixation unit and the associative network of the recognition unit.

At present, we investigate several foveation strategies in order to focus on different image features. One implemented module uses colored areas. It calculates the center of gravity for each segmented region and determines the succession of gazes. One criterion for finding a reasonable order is the color contrast between an area and its surrounding. Fig. 5.1 shows the results of this module for a typical traffic scenario. First the camera system focusses on the fixation point illustrated in the upper left of the picture and least on the fixation point in the lower right.

By the evaluation of color information we could reduce illumination effects and hence substantially improve the object recognition rate of NAVIS. A typical experiment is depicted in fig. 5.2. We shot a yellow block placed in front of an uncolored background during the different illumination conditions of a day.

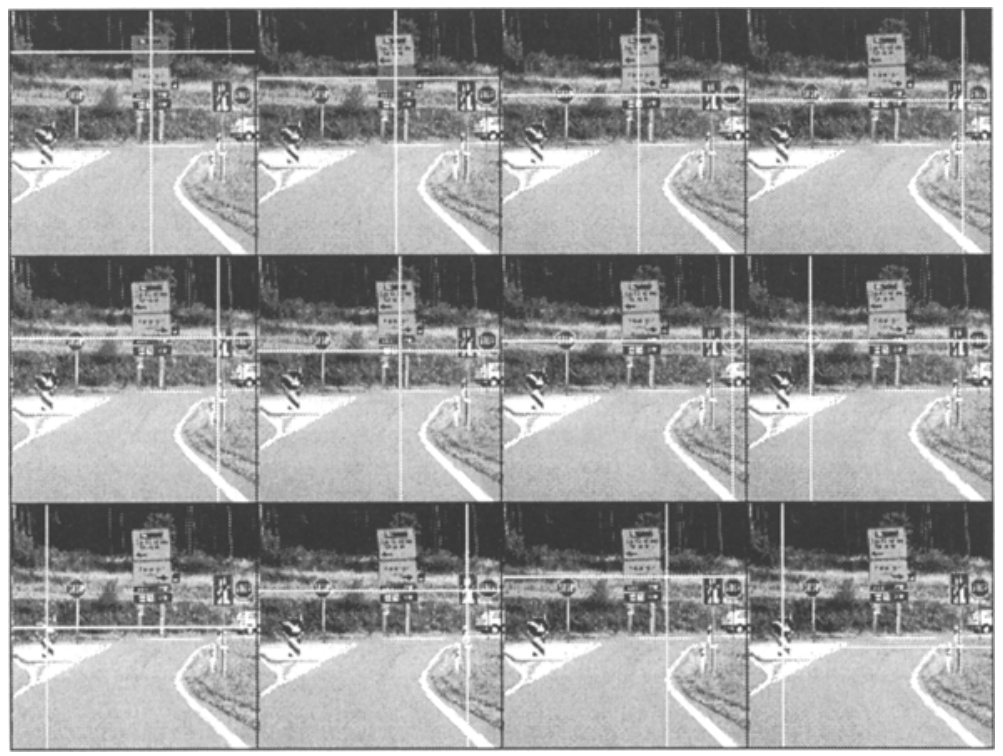

Fig. 5.1 Sequence of fixation points

Later we presented the 12-o'clock-image to our associative network which learned it in one step (primary learning). The object that has to be learnt must be presented in front of a homogenous and achromatic background. Alternatively, a figure-ground separation has to be conducted during a training phase. During the recognition stage we matched the virtual pattern of the 12-o'clock-image with all shootings of the 
yellow block. The matching formula is:

$$
\text { match }=\left.\frac{1}{I} \cdot \sum_{i=1}^{I} \sum_{j=1}^{J} \frac{f_{i, j}^{(p)}}{d_{i}^{(p)}}\right|_{\left|w_{i, j}{ }^{(1)}\right|>0,1} \cdot w_{i, j}^{(1)}
$$

where $f_{i j}^{(p)}$ are the elements of the feature vektor $f$ of the presented pattern, $d_{i}^{(p)}$ is a norm factor, and $w_{i j}{ }^{(1)}$ are the elements of the weighting vector $w^{(1)}$ of the virtual pattern.

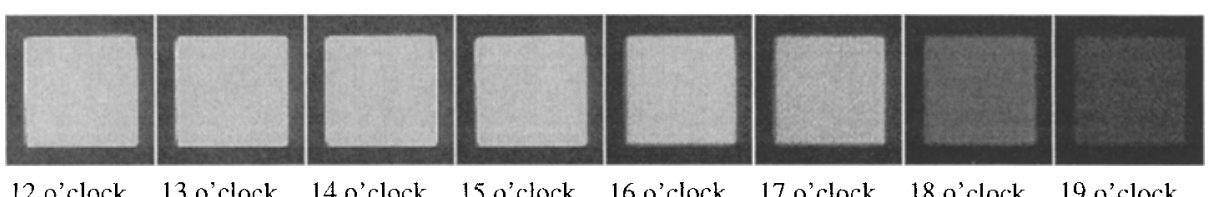

Fig. 5.2 Yellow block under varying daylight conditions

Fig 5.3 shows how the recognition capabilities of NAVIS could be improved by using the feature color. The contour based match was done by comparing illumination edges extracted from gray value images. The color based method matches the segmented areas of the opponent color channels. The gained color constancy leads to satisfactory matching results even at poor illumination conditions.

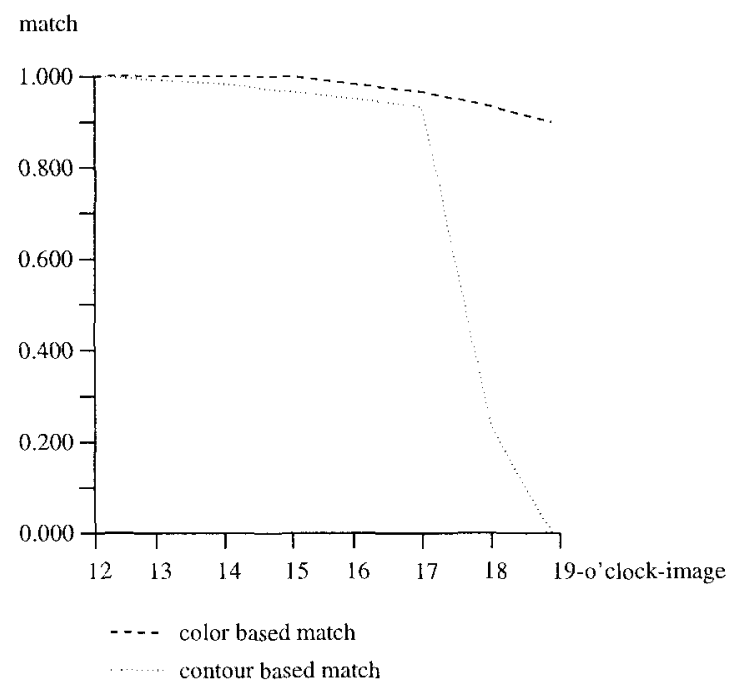

Fig. 5.3 Color based vs. contour based match 


\section{Conclusion}

In this paper we present a new opponent color model developed to extend the NeuralActive-Vision-System NAVIS. The opponent color model imitates color cells known from neurobiology. It consists of two layers, a receptor layer and a layer of simple opponent color cells (type-I-cells). The model is part of the feature extraction unit of NAVIS and serves to segment colored areas and to reduce illumination effects. Using the opponent color model we have substantially improved the fixation and recognition capabilities of NAVIS. Our current work is focussed on the modelling of double opponent color cells and the investigation of cells responding to color edges.

\section{References}

[Bollmann 1995] Bollmann, M.; Mertsching, B.; Drüe, S.: Entwicklung eines Gegenfarbenmodells für das Neuronale-Active-Vision-System NAVIS. In: Sagerer, G. (ed.): Mustererkennung 1995. Informatik aktuell. Berlin et al. (Springer) 1995, pp. $456-463+$ p. 668

[Buchsbaum 1983] Buchsbaum, G.; Gottschalk, A.: Trichromacy, opponent colours coding and optimum colour information transmission in the retina. In: Proc. Royal Society of London B, vol. 220, 1983, pp. 89-113

[Drüe 1994] Drüe, S.; Hoischen, R.; Trapp, R.: Tolerante Objekterkennung durch das Neuronale Active-Vision-System NAVIS. In: Kropatsch, W. G.; Bischof, H. (ed.): Mustererkennung 1994. Wien 1994, pp. 253-264

[Gouras 1981] Gouras, P.: Visual system 4: Color vision. In: Kandell, E. R.; Schwartz, J. H. (ed.): Principles of neural science. New York et al. (Elsevier/North-Holland) 1981, pp. 249-257

[Guth 1991] Guth, S. L.: Model for color vision and light adaption. In: Journal of the Optical Society of America A, vol. 8, no. 6, 1991, pp. 976993

[Guth 1993] Guth, S. L.: Unified model for human color perception and visual adaption II. In: Proc. SPIE: Human Vision, Visual Processing, and Digital Display 4, 1993, pp. 440-448

[Helmholtz 1867] Helmholtz, H. L. F. von: Handbuch der Physiologischen Optik. Leipzig (Voss) 1867

[Hering 1878] Hering, E.: Zur Lehre vom Lichtsinne. Berlin, 1878

[Hering 1880] Hering, E.: Zur Erklärung der Farbenblindheit aus der Theorie der Gegenfarben. In: Lotos, Jb. für Naturwiss., vol. 1, 1880, pp. 76107

[Horn 1974] Horn, B. K. P.: Determining lightness from an image. In: Computer Graphics and Image Processing, vol. 3, 1974, pp. 277299

[Horn 1986] Horn, B. K. P.: Robot vision. New York (MIT Press, McGrawHill Book Company) 1986, chapter 9: Lightness \& color, pp. 185201 
[Hubel 1968] Hubel, D. H., Wiesel, T. N.: Receptive fields and functional architecture of monkey striate cortex. In: The Journal of Physiology, vol. 195, 1968, pp. 215-243

[Hurvich 1955] Hurvich, L. M.; Jameson, D.: Some quantitative aspects of an opponents colours theory. 2. Brightness, saturation and hue in normal and dichromatic vision. In: J. Opt. Soc. Am., vol. 45, 1955, pp. 602-616

[Jameson 1955] Jameson, D.; Hurvich, L. M.: Some quantitative aspects of an opponents colours theory. 1. Chromatic responses and spectral saturation. In: J. Opt. Soc. Am., vol. 45, 1955, pp. 546-552

[Jameson 1956] Jameson, D.; Hurvich, L. M.: Some quantitative aspects of an opponents colours theory. 3. Changes in brightness, saturation and hue with chromatic adaptation. In: J. Opt. Soc. Am., vol. 46, 1956, pp. 405-415

[Land 1971] Land, E. H.; McCann, J. J.: Lightness and retinex theory. In J. Opt. Soc. Am. vol. 61, 1971, pp. 1-11

[Land 1983] Land, E. H.: Recent advances in retinex theory and some implications for cortical computations: Color vision and the natural image. In: Proc. Nat. Acad. Sci. USA, 1983, pp. 51635169

[Land 1986] Land, E. H.: An alternative technique for the computation of the designator in the retinex theory of color vision. In: Proc. Nat. Acad. Sci. USA 83, 1986, pp. 3078-3080

[Usui 1991] Usui, S.; Nakauchi, S.; Nakano, M.: Internal color representation acquired by a five-layer neural network. In: Kohonen, T.; Mäkisara, O.; Simula, O.; Kangas, J. (eds.): Artificial Neural Networks. Amsterdam et al. (Elsevier/North-Holland), vol. 1, 1991, pp. 867-872

[Usui 1994] Usui, S.; Nakauchi, S.; Miyake, S.: Acquisition of color opponent representation by a three-layered neural network model. In: Biological Cybernetics, 72, 1994, pp. 35-41

[Wiesel 1966] Wiesel, T. N.; Hubel, D. H.: Spatial and chromatic interactions in the lateral geniculate body of the rhesus monkey. In: J. Neurophysiology, vol. 29, 1966, pp. 1115-1156

[Yamaba 1993] Yamaba, K.; Miyake, Y.: Color character recognition method based on human perception. In: Optical Engineering, vol. 32, no. 1, 1993, pp. 33-40

[Young 1802] Young, T.: On the theory of light and colours. In: Philos. Trans. Royal Soc. London, 1802, pp. 12-48

[Zeki 1973] Zeki, S. M.: Colour coding in rhesus monkey prestriate cortex. In: Brain Res., vol. 53, 1973, pp. 422-427 